\title{
The quest for a staphylococcus aureus vaccine
}

\author{
Volume 2 Issue 3 - 2016 \\ Dirga Sakti Rambe,' Melvin Sanicas ${ }^{2}$ \\ 'Cipto Mangunkusumo National Referral Hospital, Indonesia \\ ${ }^{2}$ Bill and Melinda Gates Foundation, USA
}

\section{Conceptual paper}

Staphylococcus aureus is a bacterium that lives on the skin or in the nose or mouth of people (this is referred to as colonization). $S$. aureus is the cause of serious infectious diseases, such pneumonia, sepsis, toxic shock syndrome (TSS), and infective endocarditis in the United States. ${ }^{1}$ These fatal conditions associated with $S$. aureus infections depend on both cell surface and secreted virulence factors. ${ }^{2,3}$ When staph becomes resistant to commonly used antibiotics (available antibiotics are no longer effective), it is called methicillinresistant staphylococcus aureus (MRSA). ${ }^{4}$ Skin infections are very common, but infections of the bloodstream (bacteremia) and lung (pneumonia) are serious and sometimes fatal. MRSA infections are increasing in prevalence in both adults and children. The Journal of the American Medical Association ${ }^{5}$ and the Archives of Internal Medicine $^{6}$ published articles which estimated that the most virulent forms of MRSA kills up to 19,000 people every year and causes at about 7 million ER and primary-care visits each year. A vaccine that protects against fatal $S$. aureus infections is desirable to have, but a vaccine that could protect against all $S$. aureus infections, including soft tissue infections is even better.

Since the 1960s, various scientists and companies have tried to develop a staph vaccine with varying, but never complete, success. The first attempt at making a $S$. aureus vaccine was modeled on successful vaccines for Streptococcus pneumoniae and Haemophilus influenza that used antigens made up of carbohydrate molecules from the capsule that surrounds the bacterium, linked to a protein produced by another bacterium. This attempt was unsuccessful, as the recipients of the candidate vaccine developed $S$. aureus infections in the blood at the same rate as subjects who received placebo. ${ }^{8}$ Intercell and Merck Intercell, developed a vaccine candidate on an antigen (protein) that is common to almost all types of staph. Merck completed successful early clinical trials (Phases 1 and 2) in human beings and began phase 3 trials in 2009 to evaluate the safety and effectiveness of the vaccine candidate in a larger population. However, the study was stopped after experts decided that the vaccine was unlikely to show a statistically significant clinical benefit. In addition, there were worries about multi-organ dysfunction and overall mortality. ${ }^{9}$

In 2006, Vaccine Research International Plc (VRi), a small British biotech company, completed Phase 1 testing of its vaccine candidate, SA75. SA75 uses the whole staph bacterial cell 'killed' by chloroform. VRi successfully completed Phase 1 study for the treatment of hospital-acquired infections caused by staphylococcal bacteria, including drug-resistant strains such as MRSA. Should funding become available, VRi will conduct clinical trials to study the potential of SA75 to prevent staphylococcal infections in hospital patients in a larger population. ${ }^{10}$ The first version of Nabi's StaphVax consisted of antigens from the two most common staph capsules (types 5 and 8 ) and an antigen from the cell wall (type 336). Just like Intercell and VRi, the first results of Nabi's $S$. aureus vaccine were positive. In Phase 1 and 2 trials, the vaccine was safe and effective, and the developers invested significant resources to continue testing. Nonetheless, it failed to meet its primary endpoint in the confirmatory
Correspondence: Dirga Sakti Rambe, Cipto Mangunkusumo National General Hospital, Jalan Diponegoro No 7I, Senen, Jakarta Pusat, DKI Jakarta I0430, Indonesia, Email dr.dirgasakti@gmail.com

Received: February 22, 2016 | Published: June 03, 2016

Phase III clinical trial of 3,600 patients on hemodialysis. The study found no reduction in $S$. aureus types 5 and 8 infections in the StaphVAX group as compared to the placebo group. Using lessons learned from the StaphVax failure, Nabi resumed development in 2006 and began development of a modified vaccine called PentaStaph - which consists of the original StaphVax formulation plus two "virulence factors" associated with MRSA, alpha toxin and PantonValentine Leukocidin (PVL). After completion of the Phase 1 trial, PentaStaph vaccine was sold to GlaxoSmithKline Biologicals. ${ }^{11,12}$

In 2015, Pfizer Inc announced enrollment of the first patient in a Phase $2 \mathrm{~b}$ clinical trial of its investigational $S$. aureus multi-antigen vaccine in adults undergoing elective spinal fusion surgery. The study, named STRIVE (Staphylococcus aureus SuRgical Inpatient Vaccine Efficacy), aims to evaluate the safety and efficacy of the vaccine candidate and ascertain if it prevents postoperative $S$. aureus infections in patients undergoing elective spinal surgery. The phase $2 \mathrm{~b}$ study is expected to finish in March 2017 and results ready by the end of 2017. ${ }^{13}$ In 2016, Bavarian Nordic (BN) became part of a joint collaboration involving the Technical University of Denmark (DTU) and Evaxion Biotech to develop a vaccine for MRSA. The vaccine will be a Modified Vaccinia Ankara (MVA) -based immunization created with computer-based technology from Evaxion that can detect new antigens. MVA-BN is a patented vaccine platform technology of originally developed through a successful public-private partnership between Bavarian Nordic and the US Government. If the new candidate is effective and safe, human trials are expected in 2019. ${ }^{14}$

Is there a vaccine for $S$. aureus? There is no vaccine available to prevent invasive $S$. aureus disease or MRSA. Here are some of the basic research challenges that need to be addressed in order to bring us a step closer towards a $S$. aureus vaccine. ${ }^{15}$

i. S. aureus has a wide array of virulence factors that allow it to evade the host immune responses. 
ii. S. aureus strains are geographically diverse and very versatile in their antigenic repertoire.

iii. Current animal models for staphylococcal disease do not have good predictive value.

iv. Assays that can reflect physiological endpoints are needed to evaluate the host's potential to identify and eliminate $S$. aureus.

v. Certain types of antigens could or should be used to induce protective immunity. What are they?

vi. Lack of a validated clinically-relevant correlates of protection

vii. We currently do not know whether a vaccine that protects against $S$. aureus soft-tissue infection can also protect against other forms of $S$. aureus infection (i.e. bacteremia, pneumonia, and osteomyelitis).

A number of sources (US CDC, National Institute of Health, Institute of Medicine) estimate annual medical costs due to $S$. aureus infections in the USA alone to be around US\$5 billion. Several economic models consistently suggest that a $S$. aureus vaccine especially for the neonatal population would be strongly cost-effective over a wide range of vaccine efficacies, vaccine costs, and MRSA prevalence levels. ${ }^{16} S$. aureus can strike anyone with no known risk factors and can be lethal. Many areas and populations can benefit from a vaccine against $S$. aureus infection and MRSA. ${ }^{17}$

\section{Acknowledgments}

None.

\section{Conflicts of interest}

Author declares there are no conflicts of interest.

\section{Funding}

None.

\section{References}

1. Klevens RM, Morrison MA, Nadle J, et al. Invasive methicillinresistant Staphylococcus aureus infections in the United States. JAMA. 2007;298(15):1763-1771.
2. Dinges MM, Orwin PM, Schlievert PM. Exotoxins of Staphylococcus aureus. Clin Microbiol Rev. 1998;13(1):16-34.

3. Lowy FD. Staphylococcus aureus infections. $N$ Engl J Med. 1998;339(8):520-532.

4. Methicillin-resistant Staphylococcus aureus (MRSA) Infections.

5. Herold BC, Immergluck LC, Maranan MC, et al. Community-Acquired Methicillin-Resistant Staphylococcus aureus in Children With No Identified Predisposing Risk. JAMA . 1998;279(8):593-598.

6. Hersh AL, Chambers HF, Maselli JH, et al. National Trends in Ambulatory Visits and Antibiotic Prescribing for Skin and Soft-Tissue Infections. Arch Intern Med. 2008;168(14):1585-1591.

7. Salgado-Pabon W, Schlievert PM. Models matter: the search for an effectiveStaphylococcus aureus vaccine. Nature Reviews Microbiology. 2014;12:585-591.

8. Fowler VG, Proctor RA. Where does a Staphylococcus aureus vaccine stand? Clin Microbiol Infect. 2014;5:66-75.

9. Intercell announces initial results of Phase II clinical trial evaluating V710, an investigational Staphylococcus aureus vaccine, in patients with end-stage renal disease.

10. Vaccine Research International Plc Annual Report and Accounts. 2014.

11. Staphylococcus aureus vaccine conjugate--Nabi: Nabi-StaphVAX, StaphVAX. Drugs R D. 2003;4(6):383-385.

12. Jones T. StaphVAX (Nabi). Curr Opin Investig Drugs. 2002;3(1):48-50.

13. Pfizer Begins Phase 2b Study of Its Investigational Multi-antigen Staphylococcus aureus Vaccine In Adults Undergoing Elective Spinal Fusion Surgery.

14. Bavarian Nordic enters research partnership to develop MRSA vaccine.

15. Overcoming Challenges in Staph aureus Vaccine Development Meeting Summary NIAID.

16. Lee BY, Ufberg PJ, Bailey RR, et al. The Potential Economic Value of a Staphylococcus aureus Vaccine for Neonates. Vaccine. 2010;28(29):4653-4660.

17. Maranan MC, Moreira B, Boyle-Vavra S, et al. Antimicrobial resistance in staphylococci: epidemiology, molecular mechanism, and clinical relevance. Infect Dis Clin North Am. 1997;11(4):813-849. 